\title{
AD-0693 : Nailfold Capillary Abnormalities are Associated with Diabetic Nephropathy in Type 2 Diabetes
}

Hyeong Kyu Park ${ }^{1}$, Mi Kyung Kwak ${ }^{1}$, Jae Hyuk Lee ${ }^{1}$, Hye Jeong Kim ${ }^{1}$, Dong Won Byun ${ }^{1}$, Kyo Il Suh ${ }^{1}$, Myung Hi Yoo ${ }^{1}$, Hyun Sook Kim ${ }^{2}$ Division of Endocrinology and Metabolism ${ }^{1}$, Division of Rheumatology ${ }^{2}$,

Department of Internal Medicine, Soonchunhyang University College of Medicine, Seoul, Korea

Several capillary changes detected by finger nailfold capillaroscopy have been shown to correlate with microvascular complications in type 1 diabetes. However, there are few reports on the relationship between nailfold capillary abnormalities and microvascular complications in type 2 diabetes (T2DM). Herein, we investigated whether nailfold capillary microscopic changes are associated with diabetic nephropathy in patients with T2DM.

We conducted a progrssive study in patients with T2DM diagnosed within 20 years (duration of diabetes: $5.8 \pm 5.8$ years). The nailfold capillaroscopy can visualize the capillary network in fingers and is a non-invasive test. The presence of morphological abnormalities, including avascular areas, giant capillaries, dilated, tortuous, or ramified capillaries, hemorrhages and capillary architectural derangements, in finger nailfold capillaroscopy image was assessed by a single rheumatology specialist. The severity of nailfold capillary changes was scored in a semi-quantitative score $(0 \sim 3)$.

Scoring system of nailfold capillaroscopic measurements

\begin{tabular}{|l|c|c|c|c|}
\hline \multicolumn{1}{|c|}{ Scores } & $0(\mathrm{no})$ & $1(\leq 3 / \mathrm{mm})$ & $2(4-6 / \mathrm{mm})$ & $3(\geq 7 / \mathrm{mm})$ \\
\hline Architectural arrange & 0 & 1 & 2 & 3 \\
\hline Avascularity & 0 & 1 & 2 & 3 \\
\hline Giant capillaries & 0 & 1 & 2 & 3 \\
\hline Dilated capillaries & 0 & 1 & 2 & 3 \\
\hline Hemorrhages & 0 & 1 & 2 & 3 \\
\hline Tortuous capillaries & 0 & 1 & 2 & 3 \\
\hline Raminated capillaries & 0 & 1 & 2 & 3 \\
\hline
\end{tabular}

Examples of nailfold capillaroscopic score

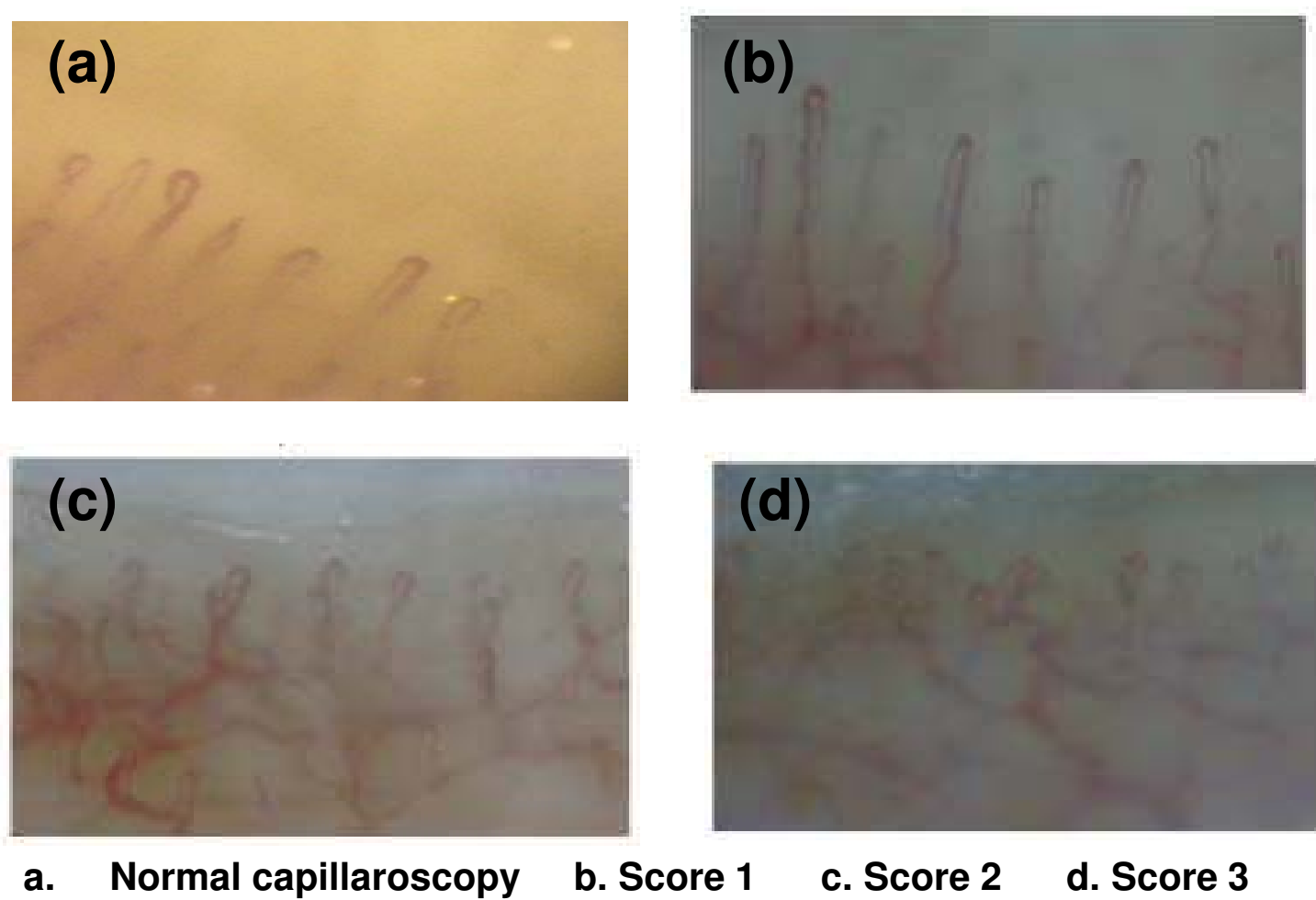

Correlations between nailfold capillaroscopic score and diabetic nephropathy in total subjects

\begin{tabular}{|c|c|c|c|}
\hline & & Architectural arrange & Avascularity \\
\hline \multirow{3}{*}{ TCR } & $r^{*} \quad(p$-value) & $0.154(0.159)$ & $0.196(0.072)$ \\
\hline & $r^{\star \star}(p$-value $)$ & $0.137(0.221)$ & $0.183(0.101)$ \\
\hline & $r^{* \star \star}(p$-value $)$ & $0.136(0.227)$ & $0.182(0.104)$ \\
\hline \multirow{3}{*}{ MCR } & $r^{*} \quad(p$-value $)$ & $\underline{0.256(0.018)}$ & $\underline{0.254(0.019)}$ \\
\hline & $r^{\star \star}(p$-value $)$ & $0.243(0.028)$ & $\underline{0.243(0.028)}$ \\
\hline & $r^{\star \star \star}(p$-value $)$ & $0.247(0.026)$ & $\underline{0.246(0.027)}$ \\
\hline \multirow{3}{*}{$\begin{array}{l}24 \text { hour urine } \\
\text { protein }\end{array}$} & $r^{*} \quad(p$-value $)$ & $0.145(0.186)$ & $0.119(0.277)$ \\
\hline & $r^{\star \star}(p$-value $)$ & $0.129(0.247)$ & $0.105(0.349)$ \\
\hline & $r^{\star \star \star}(p-$ value $)$ & $0.129(0.253)$ & $0.104(0.355)$ \\
\hline
\end{tabular}

- $\quad r^{*}=$ Partial correlation coefficient adjusted by Sex \& Age \& BMI \& DM duration

$r^{\star \star}=$ Partial correlation coefficient adjusted by Sex \& Age \& BMI \& DM duration \& Hb

$r^{\star \star \star}=$ Partial correlation coefficient adjusted by Sex \& Age \& BMI \& DM duration \& Hb \& HbA1C

Correlations between nailfold capillaroscopic score and diabetic nephropathy in subgroups according to DM duration

\begin{tabular}{|c|c|c|c|c|c|}
\hline & & \multicolumn{2}{|c|}{ Early group ( $<10$ years) } & \multicolumn{2}{|c|}{ Late group ( $\geq 10$ years) } \\
\hline & & $\begin{array}{l}\text { Architectural } \\
\text { arrange }\end{array}$ & Avascularity & $\begin{array}{l}\text { Architectural } \\
\text { arrange }\end{array}$ & Avascularity \\
\hline \multirow{2}{*}{ TCR } & r (p-value) & $\underline{0.326(0.006)}$ & $\underline{0.328(0.006)}$ & $0.161(0.386)$ & $0.178(0.337)$ \\
\hline & $\mathbf{r}^{\star}(p$-value $)$ & $\underline{0.348(0.011)}$ & $0.359(0.008)$ & $0.147(0.514)$ & $0.178(0.429)$ \\
\hline \multirow{2}{*}{ MCR } & $r \quad(p$-value) & $\underline{0.313(0.009)}$ & $\underline{0.322(0.007)}$ & $0.294(0.108)$ & $0.234(0.205)$ \\
\hline & $\mathbf{r}^{*}(p-v a l u e)$ & $\underline{0.348(0.001)}$ & $\underline{0.357(0.009)}$ & $0.306(0.166)$ & $0.224(0.317)$ \\
\hline \multirow{2}{*}{$\begin{array}{l}24 \text { hour urine } \\
\text { protein }\end{array}$} & $r \quad(p$-value $)$ & $\underline{0.344(0.007)}$ & $\underline{0.341(0.008)}$ & $0.112(0.564)$ & $0.042(0.827)$ \\
\hline & $r^{*} \quad(p-v a l u e)$ & $0.347(0.011)$ & $0.346(0.011)$ & $0.135(0.548)$ & $0.043(0.848)$ \\
\hline
\end{tabular}

$r=$ Spearman's correlation coefficient

$r^{*}=$ Partial correlation coefficient adjusted by Sex \& Age \& BMI \& DM duration \& Hb \& HbA1C 